\title{
ACÚMULO DE Cu, Mn, Ni, Pb E Zn EM LATOSSOLO VERMELHO ADUBADO COM FONTES DE LODO DE ESGOTO E CULTIVADO COM MILHO
}

\author{
Accumulation of $\mathrm{Cu}, \mathrm{Mn}, \mathrm{Ni}, \mathrm{Pb}$ AND $\mathrm{Zn}$ on a red latosol fertilized \\ with sources of sewage sludge and cultivated with corn \\ Otacilio José Passos Rangel ${ }^{2}$, Carlos Alberto Silva ${ }^{3}$ \\ Wagner Bettiol ${ }^{4}$, Luiz Roberto Guimarães Guilherme ${ }^{3}$, José Flávio Dynia ${ }^{4}$
}

\begin{abstract}
RESUMO
Realizou-se este estudo com o objetivo de avaliar o efeito das aplicações sucessivas, em doses crescentes, de duas fontes de lodo de esgoto sobre os teores totais de $\mathrm{Cu}, \mathrm{Mn}, \mathrm{Ni}, \mathrm{Pb}$ e $\mathrm{Zn}$ em solo, em três cultivos sucessivos de milho. $\mathrm{O}$ experimento foi conduzido no campo experimental da Embrapa Meio Ambiente, localizado em Jaguariúna-SP, durante os anos agrícolas 1999/2000 e 2000/2001, em Latossolo Vermelho Distroférrico. Os lodos utilizados no experimento foram obtidos nas Estações de Tratamento de Esgoto (ETEs) de Franca e Barueri. Os tratamentos, para cada fonte de lodo de esgoto, foram os seguintes: testemunha, sem adição de lodo de esgoto; NPK; aplicação de lodo de esgoto visando a suprir a quantidade de $\mathrm{N}$ normalmente requerida pelo milho; 2; 4 e 8 vezes a dose de lodo aplicada no tratamento $1 \mathrm{~N}$. As doses de lodo aplicadas (base seca) variaram de 0 a $139,24 \mathrm{Mg} \mathrm{ha}^{-1}$ para o lodo de Barueri e de 0 a 80,27 $\mathrm{Mg} \mathrm{ha}^{-1}$ para o lodo de Franca. Avaliou-se o acúmulo de $\mathrm{Cu}, \mathrm{Mn}, \mathrm{Ni}, \mathrm{Pb}$ e $\mathrm{Zn}$ pela determinação do teor total (água régia) desses metais no solo. Os teores totais de $\mathrm{Cu}$ e $\mathrm{Zn}$ em solo aumentaram com o acréscimo nas doses aplicadas das duas fontes de lodo; contudo, não foram verificados no solo teores totais dos metais pesados analisados acima dos valores estabelecidos em literatura para solos agrícolas.
\end{abstract}

Termos para indexação: Metais pesados, extrator químico, resíduo orgânico, adubação do milho.

\begin{abstract}
This study was designed to evaluate the effect of the successive applications i crescent doses, of two sources of sewage sludge on the total contents of $\mathrm{Cu}, \mathrm{Mn}, \mathrm{Ni}, \mathrm{Pb}$ and $\mathrm{Zn}$ in soil, in three successive crops of corn. The experiment was conducted in the experimental field of Embrapa Environment, located in Jaguariúna in São Paulo state over the agricultural years of 1999/2000 and 2000/2001 in a Diostroferic Red Latosol. The sludges utilized in the experiment were obtained in the Sewage Treatment Stations (STSs) of Franca and Barueri. The treatments for each source of sewage sludge were the following: control, without addition of sewage sludge; NPK; application of sewage sludge aiming to supply the amount of N normally required by corn; 2, 4 and 8 times the rate of sludge applied in the $1 \mathrm{~N}$ treatment. The rates of sludge applied (dry basis) ranged from 0 to $139.24 \mathrm{Mg} \mathrm{ha}^{-1}$ for Barueri sludge and from 0 to $80.27 \mathrm{Mg} \mathrm{ha}^{-1}$ for Franca sludge. The accumulation of $\mathrm{Cu}, \mathrm{Mn}, \mathrm{Ni}$, $\mathrm{Pb}$ and $\mathrm{Zn}$ through the determination of the total content (regia water) of these metals in soil were evaluated. The total contents of soil $\mathrm{Cu}$ and $\mathrm{Zn}$ increased with the increment of applied doses of the two sludge sources, however, were not verified in soils, total contents of the heavy metals analyzed above the values established in literature for agricultural soils.
\end{abstract}

Index terms: Heavy metals, chemical extractor, organic residue, corn fertilization.

(Recebido para publicação em 14 de abril de 2003 e aprovado em 8 de setembro de 2003)

\section{INTRODUÇÃO}

O descarte de esgotos em rios e em mananciais de água, de um modo geral, se apresenta como um dos maiores problemas ambientais a serem enfrentados nesse início de século, uma vez que o destino inadequado do esgoto produzido nas cidades brasileiras tem resul- tado em degradação acelerada das reservas de água presentes em nosso País.

A preocupação com a possibilidade de contaminação dos solos com metais pesados levou diversos países a estabelecerem limites máximos dos metais no lodo e taxa de aplicação desses no solo (USEPA, 1996; CETESB, 1999). Esse tipo de preocupação é bastante

\footnotetext{
1. Parte da dissertação de mestrado do primeiro autor, apresentada ao curso de pós-graduação em Solos e Nutrição de Plantas, Universidade Federal de Lavras/UFLA - Caixa Postal 37 - 37200-000 - Lavras, MG.

2. Pós-graduando em solos e Nutrição de Plantas - UFLA, ojpr@ufla.br

3. Professor do Departamento de Ciência do Solo da UFLA.

4. Pesquisador da Embrapa Meio Ambiente - Caixa Postal 69 - 13820-000 - Jaguariúna, SP.
} 
justificável, uma vez que o lodo, dependendo de sua origem, pode apresentar teores elevados de metais pesados, e isso pode resultar em contaminação do solo. $\mathrm{O}$ lodo proveniente de tratamento de esgoto predominantemente doméstico tem baixos teores de $\mathrm{Cd}, \mathrm{Cu}, \mathrm{Mo}$, $\mathrm{Ni}, \mathrm{Zn}, \mathrm{Pb}, \mathrm{Mn}, \mathrm{Fe}$ e Cr. Entretanto, quando os efluentes industriais predominam no esgoto, o lodo obtido pode apresentar teores de metais pesados acima da faixa permitida e, consequientemente, serem proibidos para o uso agrícola (BETTIOL et al., 1983).

A fonte do lodo de esgoto tem efeito direto no acúmulo de metais pesados em solo, principalmente quando se faz o uso continuado, em doses crescentes, nas áreas cultivadas. Aumentos nos teores totais de metais pesados em áreas adubadas com diferentes fontes e doses de lodo de esgoto foram relatadas por Mulchi et al. (1991), que verificaram, decorridos aproximadamente oito anos, elevações significativas nos teores de $\mathrm{Zn}, \mathrm{Cu}, \mathrm{Fe}, \mathrm{Pb}$, Ni e $\mathrm{Cd}$ decorrentes do aumento das doses e tipos de lodo de esgoto utilizados.

Diversos trabalhos têm demostrado que a aplicação de lodo de esgoto promove o aumento da concentração de metais no solo (BARRETO, 1995; MARQUES, 1996). Resultados obtidos por Anjos e Mattiazzo (2000), em Latossolo Roxo Distrófico que recebeu doses de lodo de esgoto que totalizaram 387,89 $\mathrm{Mg}$ ha (base seca), revelaram que mesmo com o aumento na concentração de alguns metais pesados no solo, essas elevações não ultrapassaram os limites permitidos pela USEPA (1993) e pela Diretriz da Comunidade Européia (HALL, 1998). Estudos efetuados em condições tropicais mostraram que, mesmo em condições de superdosagem, não houve acréscimo significativo no teor de metais do solo, devido à incorporação do lodo de esgoto (MIYAZAWA et al., 1994), mostrando também que nas doses mais elevadas, os metais permaneciam na superfície do solo $(0-2,5 \mathrm{~cm})$.

A simples adoção dos critérios estabelecidos nas legislações não torna segura, por tempo indeterminado, a utilização agrícola do lodo, sendo imprescindível o monitoramento periódico do solo. Estudos em campo, em longo prazo, fornecem subsídios para a elaboração de normas que padronizem o uso do lodo de esgoto em solos brasileiros, uma vez que esses estudos são escassos nas condições de clima e solos do Brasil, onde existe um grande potencial para se utilizar o lodo de esgoto em áreas agrícolas, em razão de nossos solos apresentarem baixa reserva de nutrientes e reação ácida.

Objetivou-se com este estudo avaliar o efeito de aplicações sucessivas, em doses crescentes, de dois dife- rentes lodos de esgoto sobre os teores totais de $\mathrm{Cu}, \mathrm{Mn}, \mathrm{Ni}$, $\mathrm{Pb}$ e $\mathrm{Zn}$ no solo, em três cultivos sucessivos de milho.

\section{MATERIAL E MÉTODOS}

O experimento foi conduzido no Campo Experimental da Embrapa Meio Ambiente, localizado em Jaguariúna (SP), na latitude de $22^{\circ} 41^{\prime}$ Sul, longitude $47^{\circ} \mathrm{W}$. Gr. e altitude de 570,4 m, em Latossolo Vermelho Distroférrico de textura argilosa, nos anos agrícolas de 1999/2000 e 2000/2001. Algumas características físicas e químicas do solo, analisadas seguindo-se protocolos descritos em Silva (1999), são as seguintes: matéria orgânica $=25 \mathrm{~g} \mathrm{~kg}^{-1}$; argila $=450 \mathrm{~g} \mathrm{~kg}^{-1} ; \mathrm{pH}$ em água $=4,5 ; \mathrm{Ca}^{+2}=27,5 \mathrm{mmol}_{\mathrm{c}} \mathrm{dm}^{-3}$; $\mathrm{Mg}^{+2}=8,5$ mmol $_{\mathrm{c}} \mathrm{dm}^{-3} ; \mathrm{P}$ (Mehlich-1) $=4 \mathrm{mg} \mathrm{kg}^{-1} ; \mathrm{K}^{+}=$ $59 \mathrm{mg} \mathrm{kg}^{-1}, \mathrm{Al}^{+3}=1 \mathrm{mmol}_{\mathrm{c}} \mathrm{dm}^{-3} ; \mathrm{H}+\mathrm{Al}^{+3}=35 \mathrm{mmol}_{\mathrm{c}} \mathrm{dm}^{-3}$; $\mathrm{T}=73,5 \mathrm{mmol}_{\mathrm{c}} \mathrm{dm}^{-3} \mathrm{e} \mathrm{V}=51 \%$.

Foram utilizados no estudo dois tipos de lodo de esgoto: um oriundo da Estação de Tratamento de Esgoto de Barueri, SP (Lodo de Barueri - LB), e o outro, da Estação de Tratamento de Esgoto de Franca, SP (Lodo de Franca - LF). No lodo oriundo de Barueri (SP), cidade localizada no entorno de São Paulo (SP), predominam mais os efluentes ricos em metais pesados oriundos de processos industriais, em relação ao de Franca (SP), onde as águas tratadas têm a sua origem essencialmente em domicílios. A composição química das três remessas desses lodos usadas neste estudo é apresentada na Tabela 1. Numa etapa anterior à análise química, as amostras de lodo foram secas em estufa a $65^{\circ} \mathrm{C}$ e maceradas em almofariz, sendo, a seguir, digeridas em bloco digestor de 40 provas, por meio do uso de mistura nítrico-perclórica. Os teores totais de metais pesados presentes nos lodos foram determinados por espectrometria de emissão atômica, com indução de plasma (ICP-AES), seguindo-se os protocolos analíticos descritos em Tedesco et al. (1995) para esses e para outros atributos químicos mostrados na Tabela 1 .

Os tratamentos estudados, para os lodos de Barueri (LB) e Franca (LF), foram: 1) testemunha, sem adição de lodo de esgoto (Test ${ }_{\mathrm{LB}} \mathrm{e}$ Test ${ }_{\mathrm{LF}}$ ); 2) fertilização mineral (NPK) recomendada para a cultura; 3) aplicação de lodo de esgoto visando a suprir a quantidade de $\mathrm{N}$ normalmente requerida pelo milho, ou seja, $90 \mathrm{~kg}$ ha $^{-1}$ de N (RAIJ et al., 1996) (LB1N e LF1N); 4) aplicação de duas vezes a dose de lodo descrita anteriormente (LB2N e LF2N); 5) aplicação de quatro vezes a dose de lodo listada no item 3 (LB4N e LF4N) e 6) aplicação de oito vezes a dose de lodo descrita no item 3 (LB8N e LF8N). Desse modo, as doses de lodo aplica-

Ciênc. agrotec., Lavras, v. 28, n. 1, p. 15-23, jan./fev., 2004 
das em base seca foram calculadas tendo como base o teor de nitrogênio nos lodos estudados e a necessidade de $\mathrm{N}$ do milho, segundo as recomendações da Norma $\mathrm{P}$ 4230 da CETESB (CETESB, 1999). No tratamento
NPK, utilizaram-se a fórmula 04-20-16 e uréia na adubação em cobertura. Na Tabela 2, são listadas as quantidades de lodo de esgoto e fertilizantes aplicadas nos três cultivos de milho, nos diferentes tratamentos estudados.

TABELA 1 - Características químicas dos lodos de esgoto das Estações de Tratamento de Esgoto de Barueri (LB) e Franca (LF), localizadas no Estado de São Paulo, utilizados nos três cultivos de milho.

\begin{tabular}{|c|c|c|c|c|c|c|}
\hline \multirow{2}{*}{ Atributo } & \multicolumn{2}{|c|}{ Primeiro Cultivo } & \multicolumn{2}{|c|}{ Segundo cultivo } & \multicolumn{2}{|c|}{ Terceiro cultivo } \\
\hline & LB & $\mathbf{L F}$ & LB & $\mathbf{L F}$ & LB & $\mathbf{L F}$ \\
\hline pH (água) & 5,9 & 5,2 & 5,9 & 4,9 & 5,9 & 5,5 \\
\hline Umidade $(\%)$ & 3,5 & 6,0 & 53,3 & 52,1 & 32,8 & 2,8 \\
\hline Sólidos Voláteis (\%) & 43 & 60,5 & - & - & 56,8 & 72,5 \\
\hline C orgânico $\left(\mathrm{g} \mathrm{kg}^{-1}\right)$ & 248,2 & 305,1 & 271 & 374 & 292,9 & 382,4 \\
\hline $\mathrm{N}$ - Kjeldahl $\left(\mathrm{mg} \mathrm{kg}^{-1}\right)$ & 260 & 470 & 264 & 508 & 385 & 552 \\
\hline $\mathrm{N}$ - amoniacal $\left(\mathrm{mg} \mathrm{kg}^{-1}\right)$ & 1566,9 & 4803,2 & 156 & 119 & 2401,6 & 2094,1 \\
\hline $\mathrm{N}$ - nitrato-nitrito $\left(\mathrm{mg} \mathrm{kg}^{-1}\right)$ & 106,2 & 22,0 & 106,0 & 54,8 & 51,3 & 43,9 \\
\hline$P\left(\mathrm{~g} \mathrm{~kg}^{-1}\right)$ & 15,9 & 16,0 & 31,2 & 21,3 & 26,9 & 12,9 \\
\hline $\mathrm{K}\left(\mathrm{g} \mathrm{kg}^{-1}\right)$ & 1,0 & 1,0 & 1,9 & 0,9 & 1,0 & 1,0 \\
\hline $\mathrm{Ca}\left(\mathrm{g} \mathrm{kg}^{-1}\right)$ & 24,1 & 15,4 & 26,1 & 14,5 & 27,8 & 14,5 \\
\hline $\operatorname{Mg}\left(\mathrm{g} \mathrm{kg}^{-1}\right)$ & 2,6 & 2,0 & 4,0 & 2,1 & 3,3 & 2,3 \\
\hline $\mathrm{Na}(\mathrm{g} \mathrm{kg}-1)$ & 0,3 & 0,3 & 0,6 & 0,4 & 0,4 & 0,4 \\
\hline $\mathrm{S}\left(\mathrm{mg} \mathrm{kg}^{-1}\right)$ & 13,4 & 16,3 & 10,8 & 13,3 & 17,1 & 15,7 \\
\hline Mo $\left(\mathrm{mg} \mathrm{kg}^{-1}\right)$ & 7,2 & 3,3 & 7,2 & 3,8 & 11,3 & 4,1 \\
\hline Cr total $\left(\mathrm{mg} \mathrm{kg}^{-1}\right)$ & 486,0 & 374,0 & 887,0 & 834,6 & 625,3 & 287,0 \\
\hline $\operatorname{Mn}\left(\mathrm{mg} \mathrm{kg}^{-1}\right)$ & 275,6 & 231,5 & 294,0 & 180,3 & 219,6 & 176,6 \\
\hline $\mathrm{Fe}\left(\mathrm{mg} \mathrm{kg}^{-1}\right)$ & 34933 & 28450 & 26333 & 23600 & 26600 & 23633 \\
\hline $\mathrm{Co}\left(\mathrm{mg} \mathrm{kg}^{-1}\right)$ & 12,2 & 5,0 & 10,5 & 4,9 & 9,3 & 4,8 \\
\hline $\mathrm{Ni}\left(\mathrm{mg} \mathrm{kg}^{-1}\right)$ & 333,3 & 60,5 & 467,0 & 97,5 & 336 & 68,73 \\
\hline $\mathrm{Cu}\left(\mathrm{mg} \mathrm{kg}^{-1}\right)$ & 774,0 & 178,5 & 862,0 & 216,0 & 746,0 & 214,3 \\
\hline $\mathrm{Zn}\left(\mathrm{mg} \mathrm{kg}^{-1}\right)$ & 2000,0 & 901,5 & 2895,0 & 1036,6 & 2463,3 & 1050,0 \\
\hline $\mathrm{Al}\left(\mathrm{mg} \mathrm{kg}^{-1}\right)$ & 46466 & 37000 & 35433 & 29233 & 29066 & 27866 \\
\hline $\mathrm{Cd}\left(\mathrm{mg} \mathrm{kg}^{-1}\right)$ & 7,9 & - & 8,1 & - & 6,7 & - \\
\hline $\mathrm{Pb}\left(\mathrm{mg} \mathrm{kg}^{-1}\right)$ & 115,3 & 68,7 & 90,4 & 51,1 & 78,5 & 52,7 \\
\hline $\mathrm{Ag}\left(\mathrm{mg} \mathrm{kg}^{-1}\right)$ & $<0,01$ & $<0,01$ & $<1$ & $<1$ & $<0,01$ & $<0,01$ \\
\hline As $\left(\mathrm{mg} \mathrm{kg}^{-1}\right)$ & $<1,0$ & $<1,0$ & $<1,0$ & $<1,0$ & $<1,0$ & $<1,0$ \\
\hline $\mathrm{B}\left(\mathrm{mg} \mathrm{kg}^{-1}\right)$ & 162,6 & 190,0 & 128,0 & 115,3 & 124,7 & 110,3 \\
\hline
\end{tabular}


A calagem foi realizada um mês antes do primeiro e terceiro cultivos de milho, sendo a quantidade de calcário aplicada calculada para cada parcela experimental separadamente, de forma a manter o $\mathrm{pH}$ entre 5,5 e 5,7, utilizando-se para o cálculo da necessidade de calagem o método da saturação por bases, sendo o V ideal adotado igual a $60 \%$.

Os lodos foram distribuídos em área total das parcelas experimentais e incorporados ao solo a uma profundidade de $10 \mathrm{~cm}$ com auxílio de enxada rotativa. Nas parcelas experimentais em que o lodo de esgoto foi aplicado, realizou-se, quando necessário, uma adubação com cloreto de potássio para fornecer a quantidade de K necessária para o milho (Tabela 2), em razão de o lodo ser um resíduo pobre nesse nutriente. Optou-se por não adicionar fósforo mineral nas parcelas adubadas com lodo, em razão do alto teor de $\mathrm{P}$ nesse resíduo, $\mathrm{o}$ que se mostrou acertado, uma vez que os níveis de $\mathrm{P}$ em folha estiveram dentro da faixa considerada adequada para o milho.

As cultivares de milho utilizadas no estudo foram: $1^{\circ}$ cultivo - CATI AL 30; $2^{\circ}$ cultivo- híbrido AG 1043; e $3^{\circ}$ cultivo - híbrido SAVANA 133S. O primeiro cultivo foi implantado no campo em 05/04/99; o segundo cultivo, em 13/12/99; e o terceiro cultivo, em 30/10/2000. O plantio do milho foi realizado quinze dias após a incorporação do lodo de esgoto nas parcelas experimentais, sendo os cultivos conduzidos sem irrigação. Os restos culturais foram retirados da área experimental após o término de cada ciclo de cultivo.

TABELA 2 - Quantidades de lodo de esgoto, N, $\mathrm{P}_{2} \mathrm{O}_{5}$ e $\mathrm{K}_{2} \mathrm{O}$ aplicados nos três cultivos de milho.

\begin{tabular}{|c|c|c|c|c|c|c|c|c|c|}
\hline \multirow{2}{*}{ Tratamento } & \multicolumn{3}{|c|}{$\begin{array}{c}\text { Lodo de esgoto } \\
\left(\mathrm{kg} \mathrm{ha}^{-1}-\text { base seca }\right)\end{array}$} & \multicolumn{3}{|c|}{$\begin{array}{c}\mathrm{N}, \mathrm{P}_{2} \mathrm{O}_{5}, \mathrm{~K}_{2} \mathrm{O}(04-20-16)+\mathrm{N} \\
\text { cobertura }\left(\mathrm{kg} \mathrm{ha}^{-1}\right)\end{array}$} & \multicolumn{3}{|c|}{$\begin{array}{c}\mathrm{K}_{2} \mathrm{O} \\
\left(\mathrm{kg} \mathrm{ha}^{-1}\right)\end{array}$} \\
\hline & $\begin{array}{c}1^{\circ} \\
\text { Cultivo }\end{array}$ & $\begin{array}{c}2^{\circ} \\
\text { Cultivo }\end{array}$ & $\begin{array}{c}3^{\circ} \\
\text { Cultivo }\end{array}$ & $\begin{array}{c}1^{\circ} \\
\text { Cultivo }\end{array}$ & $\begin{array}{c}\mathbf{2}^{\mathbf{0}} \\
\text { Cultivo }\end{array}$ & $\begin{array}{c}3^{\circ} \\
\text { Cultivo }\end{array}$ & $\begin{array}{c}\mathbf{1}^{\circ} \\
\text { Cultivo }\end{array}$ & $\begin{array}{c}2^{\circ} \\
\text { Cultivo }\end{array}$ & $\begin{array}{c}\mathbf{3}^{\mathbf{o}} \\
\text { Cultivo }\end{array}$ \\
\hline & \multicolumn{9}{|c|}{--Lodo Franca (LF)-- } \\
\hline Test & 0 & 0 & 0 & 0 & 0 & 0 & 0 & 0 & 0 \\
\hline $\mathrm{N}, \mathrm{P}_{2} \mathrm{O}_{5}, \mathrm{~K}_{2} \mathrm{O}$ & 0 & 0 & 0 & $400+77$ & $450+160$ & $450+182$ & 0 & 0 & 0 \\
\hline LF $1 \mathrm{~N}$ & 3014 & 3504 & 3766 & 0 & 0 & 0 & 47 & 55 & 95 \\
\hline LF $2 \mathrm{~N}$ & 6028 & 7008 & 7532 & 0 & 0 & 0 & 41 & 49 & 75 \\
\hline $\mathrm{LF} 4 \mathrm{~N}$ & 12056 & 14016 & 15064 & 0 & 0 & 0 & 28 & 39 & 30 \\
\hline LF $8 \mathrm{~N}$ & 24112 & 26032 & 30128 & 0 & 0 & 0 & 0 & 18 & 0 \\
\hline & & & & & odo Baru & (LB) - & & & \\
\hline Test & 0 & 0 & 0 & 0 & 0 & 0 & 0 & 0 & 0 \\
\hline $\mathrm{N}, \mathrm{P}_{2} \mathrm{O}_{5}, \mathrm{~K}_{2} \mathrm{O}$ & 0 & 0 & 0 & $400+77$ & $450+160$ & $450+182$ & 0 & 0 & 0 \\
\hline LB $1 \mathrm{~N}$ & 8095 & 3995 & 5315 & 0 & 0 & 0 & 55 & 47 & 65 \\
\hline LB $2 \mathrm{~N}$ & 16190 & 7990 & 10630 & 0 & 0 & 0 & 0 & 32 & 15 \\
\hline LB 4N & 32380 & 15980 & 21260 & 0 & 0 & 0 & 0 & 6 & 0 \\
\hline LB $8 \mathrm{~N}$ & 64760 & 31960 & 42520 & 0 & 0 & 0 & 0 & 0 & 0 \\
\hline
\end{tabular}

LF = lodo de esgoto da Estação de Tratamento de Esgoto (ETE) de Franca, SP; LB = lodo de esgoto da Estação de Tratamento de Esgoto (ETE) de Barueri, SP; Test = testemunha; $\mathrm{N}_{2} \mathrm{P}_{2} \mathrm{O}_{5}, \mathrm{~K}_{2} \mathrm{O}=$ adubação mineral recomendada; LF1N, LF2N, LF3N, LF4N e LF8N = doses do lodo de esgoto de Franca necessárias para fornecer uma, duas, quatro e oito vezes a quantidade de $\mathrm{N}$ da aplicação mineral; LB1N, LB2N, LB4N e LB8N = doses do lodo de esgoto de Barueri necessárias para fornecer uma, duas, quatro e oito vezes a quantidade de $\mathbf{N}$ da aplicação mineral.

Ciênc. agrotec., Lavras, v. 28, n. 1, p. 15-23, jan./fev., 2004 
Para se avaliarem os teores totais de $\mathrm{Cu}, \mathrm{Mn}, \mathrm{Ni}$, $\mathrm{Pb}$ e $\mathrm{Zn}$, foi realizada uma amostragem do solo uma semana após a aplicação das diferentes fontes e doses de lodo de esgoto, dentro da área útil das parcelas (2 linhas centrais), na camada de solo de $0-20 \mathrm{~cm}$, obtendose uma amostra composta de cinco subamostras de solo coletadas ao acaso. Na determinação dos teores totais de $\mathrm{Cu}, \mathrm{Mn}, \mathrm{Ni}, \mathrm{Pb}$ e $\mathrm{Zn}$, as amostras de solo sofreram processo de digestão de acordo com marcha analítica proposta por Nieuwenhuize et al. (1991), sendo utilizado, nessa fase, a água régia $\left(\mathrm{HCl}+\mathrm{HNO}_{3}: 1+3\right)$ como extrator. As determinações dos teores totais foram realizadas por espectrometria de emissão atômica com indução de plasma (ICP-AES).

$\mathrm{O}$ delineamento experimental utilizado foi em blocos casualizados, com três repetições, com as parcelas apresentando dimensões de 20 x $10 \mathrm{~m}$, perfazendo uma área de $200 \mathrm{~m}^{2}$.

Diante das limitações impostas pela composição química diferenciada das duas fontes de lodo de esgoto e da variação entre as doses utilizadas em cada um dos cultivos de milho, as análises estatísticas foram realizadas separadamente, o que tornou imprópria a comparação entre os resultados obtidos, optando-se pela análise e interpretação individual dos resultados referentes a cada fonte de lodo e aos cultivos de milho. O tratamento NPK não fez parte do desenho experimental deste estudo, sendo, desse modo, utilizado somente para fins de comparação com os outros tratamentos testados.

As análises de regressão dos teores totais de metais no solo, como variáveis dependentes das doses de lodo de esgoto aplicadas, foram realizadas utilizando-se o aplicativo computacional SISVAR (FERREIRA, 2000). A escolha do modelo matemático de melhor ajuste baseou-se no grau de significância da regressão e no coeficiente de determinação de cada modelo com ajuste significativo.

\section{RESULTADOS E DISCUSSÃO}

\section{Avaliação da composição química dos lodos de esgoto}

Os teores máximos de $\mathrm{Cu}, \mathrm{Mn}, \mathrm{Ni}, \mathrm{Pb}$ e $\mathrm{Zn}$ nas três remessas dos lodos de Franca (SP) e Barueri (SP), de acordo com os dados apresentados na Tabela 1, são respectivamente: 216,$0 ; 231,5 ; 97,5 ; 68,7 ; 1050,0 \mathrm{mg}$ $\mathrm{kg}^{-1}$ e 862,$0 ; 294,0 ; 467,0 ; 115,30 ; 2895,0 \mathrm{mg} \mathrm{kg}^{-1}$. Segundo Webber et al. (1984), que estabeleceram limites máximos para Mn, e CETESB (1999), os teores máximos desses metais pesados permitidos no lodo destina- do ao uso agrícola se encontram, respectivamente, dentro de intervalos ou valores de 4300, 500-3000, 420, 840, $7500 \mathrm{mg} \mathrm{kg}^{-1}$ (base seca), para $\mathrm{Cu}, \mathrm{Mn}, \mathrm{Ni}, \mathrm{Pb}$ e $\mathrm{Zn}$. Portanto, os teores dos metais pesados analisados nos lodos de esgoto de Franca e Barueri estão abaixo dos limites admitidos pela legislação, se se considerar a norma P 4230 da CETESB, que estabelece critérios para o uso agrícola de lodo de esgoto no Estado de São Paulo.

A presença de metais pesados no lodo da ETEBarueri (LB) é maior do que a verificada no lodo da ETE-Franca (LF), sendo os teores de $\mathrm{Cu}, \mathrm{Mn}, \mathrm{Ni}, \mathrm{Pb}$ e Zn no LB cerca de 3,9, 1,3, 5,0, 1,6 e 2,5 maiores do que os verificados no LF, o que reflete a natureza predominantemente doméstica dessa fonte de lodo. Assim, em relação aos teores de metais pesados, o uso agrícola do LB, comparado com o LF, requer maiores cuidados no sentido de se evitar que o solo e outras matrizes sejam contaminadas com metais pesados, principalmente por Ni e Zn, que são os metais cujos níveis no LB se encontram mais próximos das concentrações limites para lodo de esgoto estabelecidas pela CETESB. Essa maior contaminação com metais pesados do LB sinaliza para a necessidade de se identificar, no entorno do município de Barueri, indústrias ou processos industriais fontes desses metais, no sentido de que esses efluentes sejam separados dos demais, ou tratados nos locais onde são gerados, para que os teores de metais pesados em remessas do lodo da ETE-Barueri sejam diminuídos ao longo do tempo.

\section{Acúmulo de metais pesados em solo}

$\mathrm{O}$ acúmulo de metais pesados em solo, em função de aplicações sucessivas de lodo de esgoto, é um dos aspectos que causa preocupação com relação à segurança ambiental necessária para a viabilização do uso desse resíduo na agricultura. Os teores totais de metais pesados em Latossolo Vermelho Distroférrico adubado com diferentes fontes e doses de lodo de esgoto são apresentados na Figura 1. A aplicação das doses crescentes do LF não afetou significativamente os teores totais de Mn em solo em nenhum dos cultivos de milho, sendo notado somente um decréscimo de Mn em solo no $1^{\circ}$ e $3^{\circ}$ cultivos, quando se utilizou o LB. Os teores totais de $\mathrm{Cu}$ em solo aumentaram linearmente com o acréscimo nas doses do LB, não havendo efeito significativo nos teores totais desse metal em solo quando se aplicou o LF. À exceção do $1^{\circ}$ cultivo-LF, os teores totais de $\mathrm{Zn}$ em solo tiveram aumentos significativos em função da aplicação das duas fontes de lodo de esgoto. 
$\mathrm{O}$ acréscimo nos teores de $\mathrm{Cu}$ e $\mathrm{Zn}$ podem ser explicados pelas altas concentrações desses metais presentes nos lodos utilizados, sendo esse acréscimo, em geral, maior nas parcelas adubadas com o LB. Aumentos nos teores de $\mathrm{Cu}$ e $\mathrm{Zn}$ em solo, na profundidade de 0-20 cm, foram também verificados no estudo de Ligo et al. (1987), em que as doses de lodo de esgoto aplicadas atingiram até $72 \mathrm{Mg} \mathrm{ha}^{-1}$. Resultados similares foram obtidos por Berton et al. (1989), Pombo et al. (1989) e Simon et al. (1991). Em Valadares et al. (1983), em que a aplicação de lodo de esgoto foi em doses superiores a $60 \mathrm{Mg} \mathrm{ha}^{-1}$, verificou-se uma redução da produção de alface, em função da toxidez de $\mathrm{Zn}$ que atingiu teores totais no solo acima de 415 $\mathrm{mg} \mathrm{kg}^{-1}$.

Os teores totais de Ni em solo, nos três cultivos de milho, sofreram fortes acréscimos nas parcelas adubadas com o LB. Esse maior incremento nos teores totais desse elemento se explica pelo maior teor de Ni no LB $\left(378,7 \mathrm{mg} \mathrm{kg}^{-1}\right.$, média das três remessas do LB), comparado ao LF (75,6 $\mathrm{mg} \mathrm{kg}^{-1}$, média das três remessas do LF). As doses utilizadas do LF não foram suficientes para aumentar de forma significativa os teores totais de $\mathrm{Ni}$ em solo em nenhum dos cultivos de milho. Não foi observado aumento nos teores totais de $\mathrm{Pb}$ quando se utilizou o LF, o mesmo ocorrendo com o LB, à exceção do $2^{\circ}$ cultivo de milho, no qual houve um aumento linear nos teores de $\mathrm{Pb}$ em solo com o aumento das doses de lodo aplicadas.

Ao final do experimento, foram observados acréscimos nos teores totais de $\mathrm{Cu}$ em solo, em relação à testemunha, variando da menor para a maior dose de lodo de 36,4, 49,6 e 53,4\%, e 133,3, 303,8 e 245,5\% para os lodos de Franca e Barueri, respectivamente, nos três cultivos sucessivos de milho. No caso do $\mathrm{Zn}$, esses acréscimos foram de 4,7, 119,6 e $136,8 \%$, e $42,9,541,9$, e $402,7 \%$ para os mesmos lodos citados anteriormente, nos três cultivos de milho. Dessa forma, pela comparação desses valores, verifica-se o potencial de acúmulo dos metais no solo em aplicações de doses sucessivas de lodo de esgoto.

Com a sucessão dos cultivos de milho, foi verificado um decréscimo nos teores totais da maioria dos metais avaliados (Figura 1), sendo esses efeitos mais pronunciados para $\mathrm{Mn}, \mathrm{Pb}$ e $\mathrm{Zn}$. Segundo Anjos e
Mattiazzo (2001), essa queda nos teores desses metais, com o aumento do tempo de cultivo, pode estar relacionada à movimentação desses metais para camadas de solo abaixo de $20 \mathrm{~cm}$ de profundidade. É interessante notar que o decréscimo nos teores de $\mathrm{Mn}, \mathrm{Pb}$ e $\mathrm{Zn}$ ao longo do tempo foi notado para ambas as fontes de lodo, mesmo sendo as quantidades adicionadas de metais pelo LB maiores do que as supridas pelo LF.

Segundo a CETESB (2001), os valores orientadores para metais pesados em solos agrícolas são: $\mathrm{Cu}, 100 \mathrm{mg} \mathrm{kg}^{-1}$; Ni, $50 \mathrm{mg} \mathrm{kg}{ }^{-1}$; Pb, $200 \mathrm{mg} \mathrm{kg}$; e $\mathrm{Zn}, 500 \mathrm{mg} \mathrm{kg}^{-1}$. De acordo com Kabata-Pendias e Pendias (2001), a faixa ou valores críticos de metais pesados em solo são: $\mathrm{Cu}, 60-125 \mathrm{mg} \mathrm{kg}^{-1}$; Mn, 1500 $\mathrm{mg} \mathrm{kg}^{-1}$; Ni, $100 \mathrm{mg} \mathrm{kg}^{-1}$; Pb, 100-400 mg kg $70-400 \mathrm{mg} \mathrm{kg}^{-1}$. A escolha do critério para avaliação dos teores totais de metais pesados em solo foi baseada no princípio da precaução, ou seja, no critério que estabelece limites mais rígidos para metais pesados em solo. Portanto, adotaram-se os valores estabelecidos pela CETESB (2001), exceção feita ao $\mathrm{Mn}$, tendo em vista ter sido considerado o valor descrito em Kabata-Pendias e Pendias (2001).

A comparação dos valores das faixas críticas estabelecidas pelos autores acima citados com os dados apresentados da Figura 1 revela que, independentemente da dose ou fonte de lodo utilizada, não houve contaminação do solo, em relação aos níveis máximos dos elementos estudados normalmente permitidos em solo.

Quando se consideram os aspectos relacionados ao acúmulo de metais pesados em solo e os critérios que normatizam o uso agrícola de lodo, as normas estabelecidas pela USEPA (1993) e CETESB (2001) são mais permissíveis. Wallace e Wallace (1994) e McBride (1995) chamam a atenção para alguns fatores que não foram considerados pela USEPA na elaboração das normas sobre a utilização agrícola do lodo de esgoto, destacando, entre outros, aspectos relacionados com a possibilidade de interação fitotóxica entre os metais pesados, a falta de pesquisa em longo prazo, no sentido de se comprovar a segurança dos limites estabelecidos e o desconhecimento do destino desses elementos no solo após os limites máximos de acúmulo serem alcançados. 


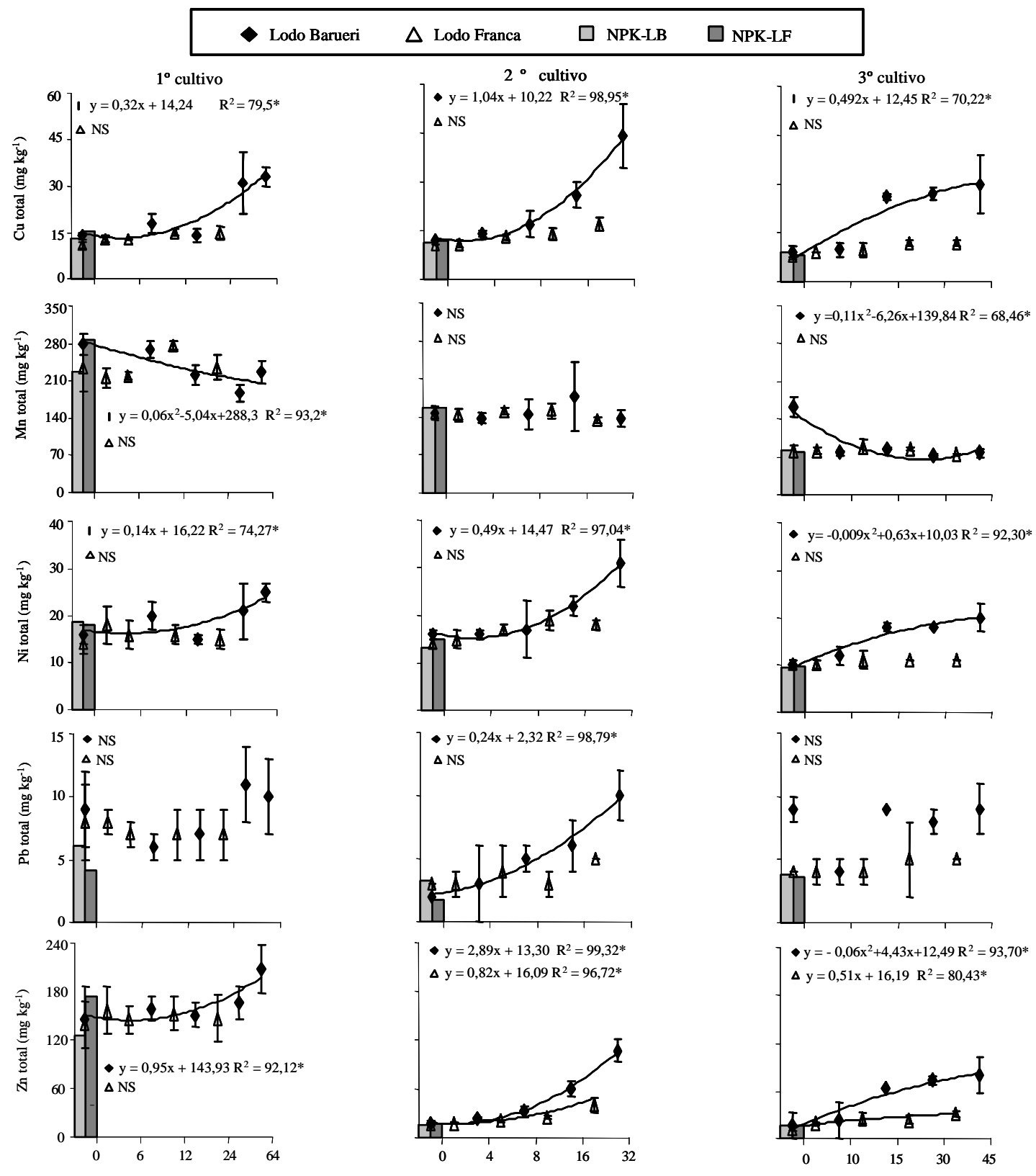

FIGURA 1 - Efeito de fontes e doses de lodo de esgoto sobre os teores totais de $\mathrm{Cu}, \mathrm{Mn}, \mathrm{Ni}, \mathrm{Pb}$ e $\mathrm{Zn}$ em Latossolo Vermelho (camada de $0-20 \mathrm{~cm}$ ) sob três cultivos sucessivos de milho (* significativo pelo teste $\mathrm{F}$ a $5 \%$ de probabilidade; NS - não-significativo). 


\section{CONCLUSÕES}

a) A aplicação de lodo de esgoto aumentou os teores totais de $\mathrm{Cu}, \mathrm{Ni}$ e $\mathrm{Zn}$ em solo, sendo os maiores acréscimos verificados nas áreas adubadas com o lodo de Barueri.

b) Houve diminuição nos teores totais dos metais pesados em solo com o cultivo seqüencial de milho.

c) Não ocorreu acúmulo de metais pesados em solo acima dos limites críticos estabelecidos em literatura.

\section{AGRADECIMENTOS}

À Embrapa, representada pelos seguintes Centros de Pesquisa: Embrapa Solos e Embrapa Meio Ambiente, pelo financiamento do estudo (Projeto SEP número 11.1999.237), em especial ao Dr. Celso Vainer Manzatto, Marcelo Saldanha e Viviane Scaleira, pelo apoio às ações de pesquisa deste estudo e pela ajuda nas análises laboratoriais, e ao José Abraão e Dra. Rita C. Boeira, pela manutenção e condução do experimento em campo e ajuda constante na coleta e preparo de amostras de lodo, solo, folhas e grãos de milho.

\section{REFERÊNCIAS BIBLIOGRÁFICAS}

ANJOS, A. R. M. dos; MATTIAZZO, M. E. Extratores para $\mathrm{Cd}, \mathrm{Cu}, \mathrm{Cr}, \mathrm{Mn}, \mathrm{Ni}, \mathrm{Pb}$ e $\mathrm{Zn}$ em latossolos tratados com biossólido e cultivado com milho. Scientia Agrícola, Piracicaba, v. 58, n. 2, p. 337-344, abr./jun. 2001.

ANJOS, A. R. M. dos; MATTIAZZO, M. E. Metais pesados em planta de milho cultivadas em latossolo repetidamente tratado com biossólido. Scientia Agrícola, Piracicaba, v. 57, n. 4, p. 769-776, out./dez. 2000.

BARRETO, M. C. V. Degradação da carga orgânica de diferentes resíduos e seus respectivos efeitos em alguns atributos químicos e físicos do solo. 1995. 106 f. Dissertação (Doutorado em Agronomia) - Escola Superior de Agricultura "Luiz de Queiroz", Universidade de São Paulo, Piracicaba, 1995.

BERTON, R. S.; CAMARGO, O. A.; VALADARES, J. M. A. S. Absorção de nutrientes pelo milho em resposta à adição de lodo de esgoto em cinco solos paulistas. Revista Brasileira de Ciência do Solo, Campinas, v. 13, n. 2, p. 187-192, maio/ago. 1989.
BETTIOL, W.; CARVALHO, P. C. T.; FRANCO, B. J. D. C. Utilização do lodo de esgoto como fertilizante. $\mathbf{O}$ Solo, Piracicaba, v. 75, n. 1, p. 44-54, jan./jun. 1983.

COMPANHIA DE TECNOLOGIA DE SANEAMENTO AMBIENTAL - CETESB. Aplicação de biossólidos de sistemas de tratamento biológico em áreas agrícolas: critérios para projeto e operação: norma P 4230. São Paulo, 1999.

COMPANHIA DE TECNOLOGIA DE SANEAMENTO AMBIENTAL - CETESB. Relatório de estabelecimento de valores orientadores para solos e água subterrâneas do Estado de São Paulo. São Paulo, 2001. 232 p.

FERREIRA, D. F. Análises estatísticas por meio do Sisvar para Windows 4. 0. In: REUNIÃO ANUAL DA REGIÃO BRASILEIRA DA SOCIEDADE INTERNACIONAL DE BIOMETRIA, 45., 2000, São Carlos. Anais... São Carlos, SP: UFSCAR, 2000. p. 255-258.

HALL, J. E. Standardizing and the management of biossolids : the international experience. In: SEMINÁRIO SOBRE GERENCIAMENTO DE BIOSSÓLIDOS DO MERCOSUL, 1., 1998, Curitiba. Anais... Curitiba: Companhia de Saneamento do Paraná/Associação Brasileira de Engenharia Sanitária e Ambiental, 1998. p. 113-122.

KABATA-PENDIAS, A.; PENDIAS, H. Trace elements in soils and plants. 3. ed. Boca Raton: CRC, 2001. 413 p.

LIGO, M. A. M.; TEDESCO, M. J.; SIMON, Z.; GIANELLO, C. Efeito do lodo ativado no sistema soloplanta-água- $1^{\circ}$ cultivo. In: CONGRESSO BRASILEIRO DE CIÊNCIA DO SOLO, 21., 1987, Campinas. Resumos... Campinas: SBCS, 1987. p. 112-113.

MARQUES, M. O. Incorporação de lodo de esgoto em solos cultivados com cana-de-açúcar. 1996. $111 \mathrm{f}$. Tese (Livre Docência) - Faculdade de Ciências Agrárias e Veterinárias, Universidade Estadual Paulista "Julio de Mesquita Filho”, Jaboticabal, 1996.

McBRIDE, M. B. Toxic metal accumulation from agricultural use of sludge: are USEPA regulations protective? Journal of Environmental Quality, Madison, v. 24, n. 1, p. 5-18, Jan./Feb. 1995. 
MIYAZAWA, M.; YAMASHITA, M.; PAVAN, M. A.; OLIVEIRA, E. L. Dinâmica do Mn do solo e absorção pela planta. In: REUNIÃO BRASILEIRA DE FERTILIDADE DO SOLO E NUTRIÇÃO DE PLANTAS, 21., 1994, Petrolina. Anais... Petrolina: SBCS, 1994. p. 184-185.

MULCHI, C. L.; ADAMU, C. A.; BELL, P. F.; CHANEY, R. L. Residual heavy metal concentrations in sludge-amended coastal plain soils: I. comparison of extractants. Communications in Soil Science and Plant Analysis, New York, v. 22, n. 9/10, p. 919-941, 1991.

NIEUWENHUIZE, J.; POLEY-VOS, C. H.; AKKER, A. van den; DELFT, A. van. Comparison of microwave and convention extraction techniques for the determination of metals in soil, sediment and sludge samples by atomic spectrometry. Analyst, Dordrecht, v. 116, p. 347-351, 1991.

POMBO, L. C. A.; TEDESCO, M. J.; GIANELlO, C. Descarte de lodo de curtume em solo podzólico vermelho-amarelo. In: CONGRESSO BRASILEIRO DE CIÊNCIA DO SOLO, 23., 1989, Porto Alegre. Resumos... Porto Alegre: SBCS, 1989. p. 309.

RAIJ, B. van; CANTARELLA, H.; QUAGGIO, J. A.; FURLANI, A. M. C. Recomendações de adubação e calagem para o Estado de São Paulo. Campinas: IAC, 1996. 285 p.

SILVA, F. C. (Ed.). Manual de análises químicas de solos, plantas e fertilizantes. Brasília: EMBRAPAComunicação para Transferência de Tecnologia, 1999. $370 \mathrm{p}$.
SIMON, Z.; TEDESCO, M. J.; GIANELLO, C. Long term land application of activated sludge from petrochemical wastewater treatment plant. Water Science and Technology, Oxford, v. 24, n. 11, p. 19-31, 1991.

TEDESCO, M. J.; GIANELLO, C.; BISSANI, C. A.; BOHEN, H.; VOLKWEISS, S. J. Análise de solo, plantas e outros materiais. Porto Alegre: UFRGSDepartamento de Solos, 1995. 174 p.

\section{UNITED STATES ENVIRONMENTAL} PROTECTION AGENCY. Standards for the use or disposal of sewage sludge: 40CFR. Washington, 1996. $152 \mathrm{p}$.

UNITED STATES ENVIRONMENTAL PROTECTION AGENCY. 40 CFR - Part 503. Standards for the use and disposal of sewage sludge. Federal Region, Washington, v. 58, p. 9387-9415, 1993.

VALADARES, J. M. A. S.; GAL, M.; MILGELGRIN, V.; PAGE, A. I. Some heavy metals in soils treated with sewage sludge, their effects on yield, and their uptake by plants. Journal of Environmental Quality, Madison, v. 12, n. 1, p. 49-57, Jan./Feb. 1983.

WALLACE, A.; WALLACE, G. A. A possible flaw in EPA'S 1993 new sludge rule due to heavy metal interaction. Communications in soil science and plant analysis, New York, v. 25, n. 1/2, p. 129-135, 1994.

WEBBER, M. D.; KLOKE, A.; TJELL, J. C. A review of current sludge use guidelines for the control of heavy metal contamination in soils. In: L'HERMITE, P.; OTT, H. Processing and use of sewage sludge. 3. ed. Dordrecht: Papers, 1984. p. 371-386. 